\title{
Dental school will only admit applicants with 'Hollywood smiles'
}

The University of Yorkshire has recently announced that it will only consider applicants with Hollywood smiles to be the students of its dental school.

This evaluation will form part of its admission process starting in the year 2021, in addition to multiple mini interviews (MMI) and biomedical admission test (BMAT).

Joanne Davies, the dean of the dental school, said: 'Applicants must demonstrate to us that not only they are dentally fit, they must also have aesthetically pleasing smiles to be our dental students.'

Among the criteria that will be looked at in the dental evaluation are facial symmetry, smell of breath, shades of their teeth, alignment of the teeth, number of teeth and number of restorations.

The school is working with a panel of aesthetic experts to perform the aesthetics evaluation. The dental evaluations will be conducted and assessed by staff and clinical tutors themselves. This will allow the tutors to gauge the oral hygiene conditions of their potential students and decide if they are dentally fit to be one.

When asked the reason for this move, Davies explained: 'We hope to only admit applicants with the desirable Hollywood smiles as we believe that this will restore the patients' confidence in our dental students especially when they are treating them during their undergraduate studies. I cannot emphasise enough the importance of this aspect.

'Everyone wants to be treated by someone who is not only smart and good in communication, but also has smile that is pleasing to the eyes. I believe this will boost the patients' compliance to oral hygiene instructions provided and encourage more patients to seek treatments at our dental school.'

By Othman Zuhir 